\title{
Study of exclusive processes within the GPD approach
}

\author{
Sergey Goloskokov ${ }^{1, *}$ \\ ${ }^{1}$ Bogoliubov Laboratory of Theoretical Physics, Joint Institute for Nuclear Research, Dubna 141980, \\ Moscow region, Russia
}

\begin{abstract}
In this report, we analyze the exclusive meson production in leptonhadron and hadron processes to study Generalized Parton Distributions (GPDs). It is shown that in both reactions, the photoproduction mechanism of vector meson (VM) production predominates. We estimate the VM production cross section and spin observables.

The possibility to study the exclusive VM production at NICA was analyzed. One can investigate different observables in $\rho, \omega$ and $J / \Psi$ productions to study quark and gluon GPDs. The other important process is the exclusive Drell-Yan (D-Y) production in the proton-proton reaction determined by two GPDs contributions. This reaction gives an important test of the GPD model and hopefully can be studied at NICA energies.
\end{abstract}

\section{Introduction}

In this report, we analyze what exclusive processes can be used to extract GPDs. GPDs contain an extensive information about the hadron structure. In the forward limit, GPDs are equal to parton distribution functions (PDFs). The GPDs moments are connected with hadron form factors and contain the information on the parton angular momenta (see e.g. review [1]).

An important process to study GPDs is the meson leptoproduction at high photon virtuality where the handbag approach was successfully used. In this approach, the amplitudes factorize into the hard subprocess and GPDs [2]. The handbag approach was applied to the light VM [3] and the pseudoscalar meson leptoproduction [4]. Some results for the cross section and spin observables are shown in Sec. 2. At intermediate $Q^{2}$, the essential role is played by the twist- 3 transversity effects. In this section, we discuss the transversity effects in the VM production [5]. The transversity contributions to the $\pi^{0}$ production were analyzed in [6].

In Sec. 3, we investigate a possibility to study the VM production at hadron accelerators like the NICA Collider in Dubna. It is shown that $\rho, \omega$ and $J / \Psi$ productions can be analyzed via a photoproduction mechanism like lepton-proton reactions.

In Sec. 4, we study the exclusive D-Y process in the proton-proton reaction proposed in [7], which is determined by two GPDs contributions. The first preliminary estimations of this reaction at NICA energies were made.

\footnotetext{
*e-mail: goloskkv@ theor.jinr.ru
} 


\section{Handbag approach. Properties of meson leptoproduction}

Within the handbag approach, the meson production amplitude at high $Q^{2}$ is factorized [2] into the hard subprocess amplitude $\mathcal{H}$ and GPDs $F$, Fig. 1 . The leading contributions to the proton non-flip and spin-flip amplitudes can be expressed in terms of various parton effects

$$
\mathcal{M}_{\mu^{\prime}+, \mu+} \propto \Sigma_{a} \int_{-1}^{1} d x \mathcal{H}_{\mu^{\prime}+, \mu+}^{a} F^{a}(x, \xi, t) ; M_{\mu^{\prime}-, \mu+} \propto \frac{\sqrt{-t}}{2 m} \Sigma_{a} \int_{-1}^{1} d \bar{x} \mathcal{H}_{\mu^{\prime}+, \mu+}^{a} E^{a}(\bar{x}, \xi, t) .
$$

Here $a$ is a flavor factor. In the VM production, we have in $F^{a}, E^{a}$ GPDs contributions for gluons, valence and sea quarks. Polarized GPDs $\tilde{F}^{a}$ contribute to the PM production.

The subprocess amplitude is calculated within the modified perturbative approach [8]. We consider the power $k_{\perp}^{2} / Q^{2}$ corrections in the propagators of the hard subprocess amplitude $\mathcal{H}$ together with the nonperturbative $\mathbf{k}_{\perp}$-dependent meson wave function [9]. The power corrections can be regarded as an effective consideration of the higher twist effects. The gluonic corrections are treated as the Sudakov factors.

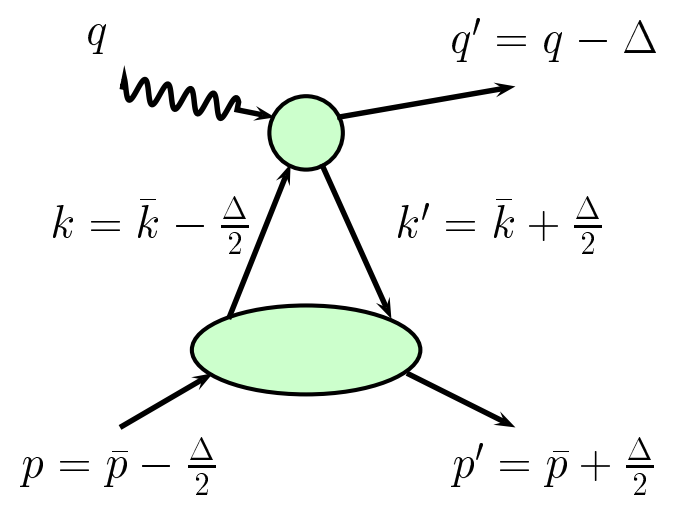

Figure 1. The handbag diagram for the meson photoproduction off proton

The GPDs are estimated using the double distribution representation [10], which connects GPDs $F$ with PDFs $h$ through the double distribution function $\kappa$. For the valence quark contribution it looks like

$$
\kappa_{i}(x, y, t)=h_{i}(x, t) \frac{3}{4} \frac{\left[(1-|x|)^{2}-y^{2}\right]}{(1-|x|)^{3}} .
$$

The $t$-dependence in PDFs $h$ is considered in the Regge form

$$
h(\beta, t)=N e^{b_{0} t} \beta^{-\alpha(t)}(1-\beta)^{n},
$$

and $\alpha(t)$ is the corresponding Regge trajectory. The parameters in (3) are obtained from the known information about PDFs [11] or from the nucleon form factor analysis [12].

The $Q^{2}$ dependencies of the $\rho$ cross section at HERA are shown in Fig. 2 (left [3]). The leading twist results are presented by the dashed line in Fig. 2 . One can see that the $k_{\perp}^{2}$ corrections essentially suppress the cross section at low $Q^{2}$.

The model results reproduce well the energy dependence of the $\phi$ production cross section from CLAS to HERA energies [3]. This means that the gluon and sea quark effects, which are essential for $\phi$ production, are well reproduced. However, the cross section falls at energies 

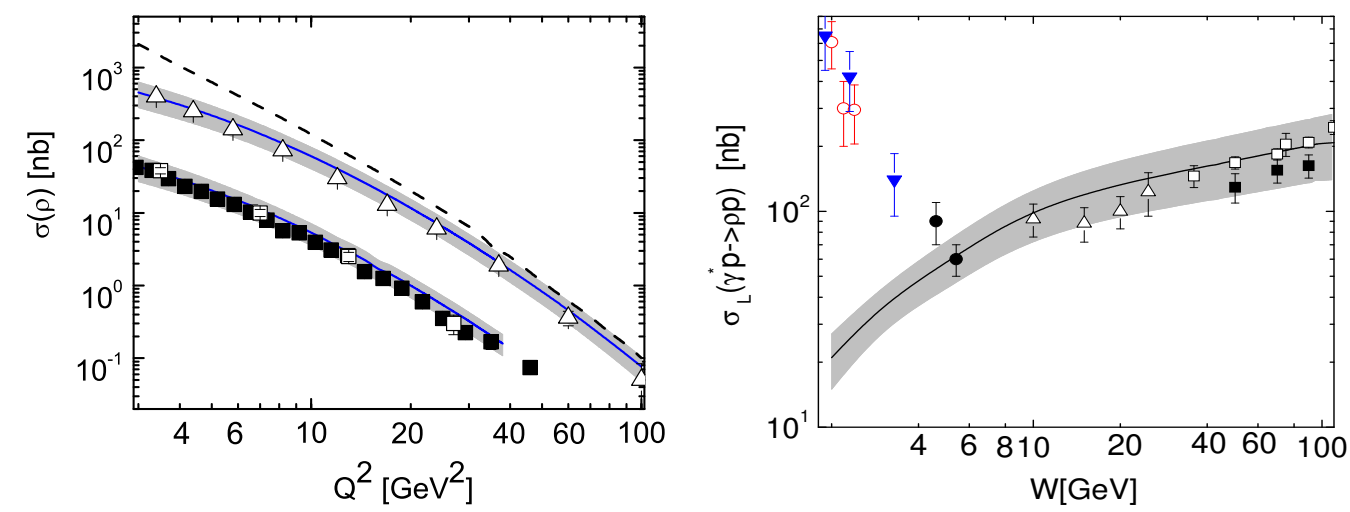

Figure 2. Left: The $Q^{2}$ dependence of the cross sections for the $\rho$ production at $W=75 \mathrm{GeV} / 10$ and $W=90 \mathrm{GeV}$. The dashed line shows the leading twist results. Right: The longitudinal cross section for the $\rho$ production at $Q^{2}=4.0 \mathrm{GeV}^{2}$
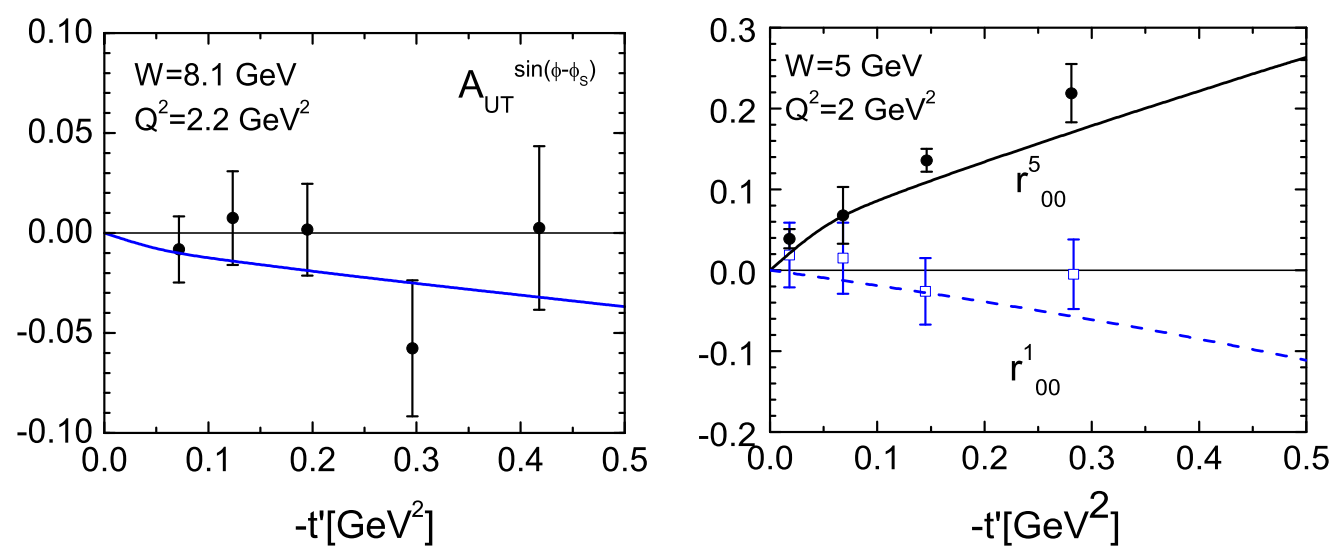

Figure 3. Left: The $t^{\prime}$ dependence of the $A_{U T}^{\sin \left(\phi-\phi_{s}\right)}$ asymmetry for the $\rho$ production at COMPASS. Right: The transversity effects in SDME for the $\rho$ production at HERMES

$W \leq 5 \mathrm{GeV}$ for $\rho$ production. This is in contradiction with the CLAS results [13] that show an essential increase of the cross section in this energy range, Fig 2 (right). This means that we have a problem only with the valence quark contribution at low energies.

Above we consider the leading twist contributions to the VM production amplitudes. It was found that at low $Q^{2}$ we should also take into account the contributions from the transversity GPDs $H_{T}$ and $\bar{E}_{T}$, which determine the amplitudes $M_{0-,++}$ and $M_{0+,++}$, respectively. Within the handbag approach, the transversity GPDs are accompanied by the twist- 3 meson wave function in the hard amplitude $\mathcal{H}$ [6], which is the same for both $\mathcal{M}_{0 \pm,++}^{t w-3}$ amplitudes in 
(4)

$$
\mathcal{M}_{0-,++}^{t w-3} \propto \int_{-1}^{1} d \bar{x} \mathcal{H}_{0-,++}(\bar{x}, \ldots) H_{T}(\bar{x}, \ldots) ; \mathcal{M}_{0+,++}^{t w-3} \propto \frac{\sqrt{-t^{\prime}}}{4 m} \int_{-1}^{1} d \bar{x} \mathcal{H}_{0-,++}(\bar{x}, \ldots) \bar{E}_{T}(\bar{x}, \ldots) .
$$

The details of the calculations and transversity GPDs parameterization can be found in [5].

In Fig. 3 (left), the $A_{U T}^{\sin \left(\phi-\phi_{s}\right)}$ asymmetry at the COMPASS energy is shown. It is determined by

$$
A_{U T}^{\sin \left(\phi-\phi_{s}\right)} \sim \operatorname{Im}\left[M_{0-, 0+}^{*} M_{0+, 0+}-M_{0-,++}^{*} M_{0+,++}\right] .
$$

Here the essential contribution comes from the first term $\propto<H>\langle E\rangle$.

The SDMEs which are determined by the $<\bar{E}_{T}>$ effects at HERMES are shown in Fig. 3 (right). They look as follows:

$$
r_{00}^{5} \sim \operatorname{Re}\left[M_{0+, 0+}^{*} M_{0+,++}\right] ; \quad r_{00}^{1} \sim-\left|M_{0+,++}\right|^{2} .
$$

These SDMEs will be equal to zero if the transversity effects are absent. A good description of the cross section and spin observables shows that we understand correctly the properties of the leading twist contributions and transversity effects.

\section{GPDs in vector meson production at NICA}

In this section, we analyze a possibility to study the meson production in hadron reactions. Here we discuss NICA Collider facilities [14]. The NICA detectors should have a detailed determination of final particles. Then it is possible to detect an exclusive reaction like $p p \rightarrow p p M$ where $M$ is a meson. The reaction when the proton radiates a hard photon with virtuality $Q^{2}$ that interacts with the other proton and produces the meson $M$ is shown in Fig. 4. The photon momenta and virtuality $Q^{2}$ can be found from the final proton angle and momenta. This photoproduction contribution is similar to the corresponding process in the lepton-proton reaction. However, in the proton-proton reaction we should have the strong interaction contribution in addition to the photon exchange. Estimations show that at NICA the energy $s_{1}=\left(p_{1}^{\prime}+q^{\prime}\right)^{2}$ is rather large $s_{1} \geq 100 \mathrm{GeV}^{2}$. At such energies, the gluon contribution should predominate [3]. The minimum number of gluons which can contribute to the VM production process is equal to 3. This exchange can be associated with the Odderon contribution. So we have at NICA the photon contribution and the Odderon effects in the VM production, Fig. 4. Generally, the Odderon contribution is rather small in most reactions and was not observed experimentally. Thus, the photon channel should be dominated in the VM production and we can use our estimations from $\operatorname{Sec} 2$.

At NICA, we shall have the energy in the $\gamma p$ system $W=\sqrt{s_{2}=(q+p)^{2}} \sim 5-15 \mathrm{GeV}$. The light vector meson production $M=\rho, \omega$ can be studied at $Q^{2} \sim 3-4 \mathrm{GeV}^{2}$. For such $Q^{2}$ and $W \sim 10 \mathrm{GeV}$ the photon channel cross section is about $\sigma_{\rho} \sim 100 \mathrm{nb}$ and $\sigma_{\omega} \sim 20 \mathrm{nb}$ [3], Fig. 2. The cross section is rather large and one can obtain information on the GPDs $H$ for gluon, sea and valence quark distributions from the light VM production. The studies using longitudinally and transversely proton polarization at NICA can give the information on spin asymmetries in the meson production [3]. For a transversely polarized proton beam, the information on the GPD $E$ can be obtained from the asymmetry $A_{U T}$ [3], Fig. 3. The GPDs $H$ and $E$ contribute to the Ji sum rule [15]

$$
J^{q}=\frac{1}{2} \int x d x\left(H^{q}(x, \xi, 0)+E^{q}(x, \xi, 0)\right),
$$

which determines proton angular momenta. This study will shed light on solution of the socalled Spin Crises. This will give an important information on GPDs. If the results from 


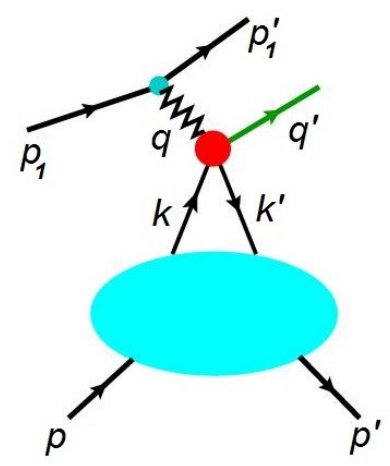

Figure 4. Meson production in hadron reactions. The zigzag line shows the gluon and the strong interaction contribution (Odderon, see the text)

the proton-proton scattering are different from the lepton-proton reaction, one can test the Odderon contribution to the VM production process.

The $J / \Psi$ production can be studied at $W \sim 15 \mathrm{GeV}$ and $Q^{2} \sim 2 \mathrm{GeV}^{2}$. The large meson mass allows one to perform the perturbative calculation at a sufficiently low $Q^{2}$. If $Q^{2}$ is not large, the photon exchange in $J / \Psi$ production should definitely dominate. The cross section in the photon channel is about $\sigma_{J / \Psi} \sim 10 \mathrm{nb}$. This process is determined by the gluon GPDs $H^{g}$. The $\tilde{H}^{g}$ effects can be tested in the $A_{L L}$ asymmetry of the $J / \Psi$ production in polarized reactions at NICA.

\section{Exclusive Drell-Yan process with two GPDs contribution}

There is another possibility to study GPDs in the proton-proton reaction. In [7], there was proposed the exclusive D-Y production in the proton-proton reaction determined by two GPDs. In the final state, we should observe two protons and a pair of leptons. An example of the process that contains the gluon and quark contribution is shown in Fig. 5.
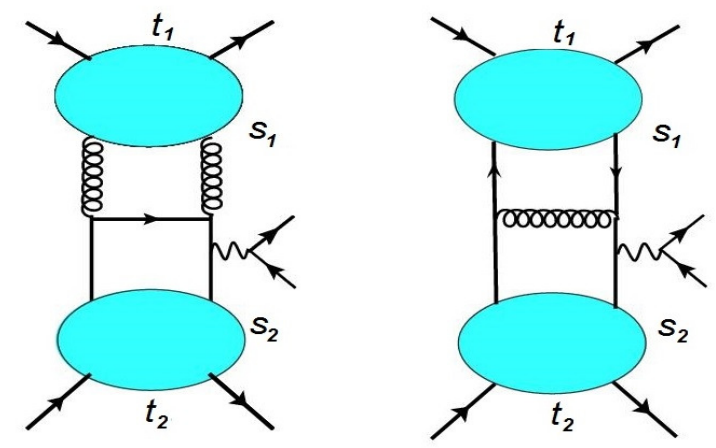

Figure 5. Drell-Yan process with gluon and quark GPDs 
The study of the cross section of the exclusive D-Y reaction is in progress now [16]. It is shown that the gluon and quark sea GPDs lead to the cross section which does not decrease with energy and this reaction can be studied at high energies.

We estimate the cross section at NICA energies $\sqrt{s}=24 \mathrm{GeV}$ and $Q^{2}=5 \mathrm{GeV}^{2}$ integrated over $s_{1}$ and $s_{2}$ (see Fig. 5). We have preliminary results for $d \sigma /\left(d Q^{2} d t_{1} d t_{2}\right)$. It is found that the cross section of the exclusive D-Y process is equal to zero at $t_{1}=0, t_{2}=0$. It has a strong maximum at a small momentum transfer where the cross section is about $40-50$ $\mathrm{pb} / \mathrm{GeV}^{6}$. At higher $-t_{1},-t_{2}$, the cross section decreases exponentially. Integrated over $t_{1}, t_{2}$, the $\mathrm{D}-\mathrm{Y}$ cross section $d \sigma / d Q^{2} \sim 3 \mathrm{pb} / \mathrm{GeV}^{2}$. This result is smaller with respect to the cross section of the inclusive D-Y reaction because the considered contribution, Fig. 5, is a part of the inclusive process. The exclusive D-Y cross section is rather small, although we hope to be able to observe it at NICA. This gives an opportunity to study GPDs at NICA in the exclusive D-Y production.

\section{Conclusion}

In this report, we analyze the meson photoproduction as an important mechanism to study GPDs. The handbag approach where the amplitudes factorize into the hard subprocess and GPDs was successfully used for the hard VM production to study the GPDs $H$. In the polarized case, we have an access to the GPDs $E$ from asymmetries. The transversity effects can be studied in some SDMEs in the VM production.

We discuss a possibility to analyze the VM production in hadron reactions at NICA. It is found that in addition to the photoproduction mechanism the Odderon contribution is possible, which is expected to be small. In the light $\rho, \omega \mathrm{VM}$ productions at NICA we can test the $H, \tilde{H}$ and $E$ GPDs contributions. The $J / \Psi$ production will give the information about the gluon GPDs $H^{g}, \tilde{H}^{g}$. The exclusive D-Y process with the double GPDs contribution is an important test of the GPD model. The D-Y cross section is rather small, but hopefully might be analyzed.

Thus, an important information on the GPDs structure can be obtained at NICA with polarized beams.

\section{References}

[1] M. Diehl, Phys. Rept. 388, 41 (2003)

[2] X. Ji, Phys. Rev. D 55, 7114 (1997). A. V. Radyushkin, Phys. Lett. B 380, 417 (1996).

J. C. Collins et al., Phys. Rev. D 56, 2982 (1997)

[3] S. V. Goloskokov and P. Kroll, Euro. Phys. J. C 42, 281-301 (2005). ibid C 50, 829 (2007). ibid C 53, 367 (2008). ibid C 59, 809-819 (2009)

[4] S. V. Goloskokov and P. Kroll, Euro. Phys. J. C 65, 137 (2010)

[5] S. V. Goloskokov and P. Kroll, Euro. Phys. J. C 74, 2725 (2014)

[6] S. V. Goloskokov and P. Kroll, Euro. Phys. J. A 47, 112 (2011)

[7] O. V. Teryaev, PoS QNP, 055 (2012). A. A. Pivovarov and O. V. Teryaev, AIP Conf. Proc. 1654, 070008 (2015)

[8] J. Botts and G. Sterman, Nucl. Phys. B 325, 62 (1989)

[9] R. Jakob and P. Kroll, Phys. Lett. B 315, 463 (1993). J. Bolz, J. G. Körner and P. Kroll, Z. Phys. A 350, 145 (1994)

[10] I. V. Musatov and A. V. Radyushkin, Phys. Rev. D 61, 074027 (2000)

[11] J. Pumplin et al., JHEP 0207, 012 (2002) 
[12] M. Diehl, T. Feldmann, R. Jakob, and P. Kroll, Eur0. Phys. J. C 39, 1 (2005)

[13] J. P. Santoro et al. [CLAS Collab.], Phys. Rev. C78,025210 (2008).

[14] V. D. Kekelidze et al. Eur. Phys. J. A 52, 211 (2016)

[15] X. Ji, Phys. Rev. Lett. 78, 610 (1997)

[16] S. V. Goloskokov, P. Kroll and O. V. Teryaev, in progress 